\section{Cahiers de Narratologie}

Analyse et théorie narratives

29 | 2015

Street Art 1

\title{
Keith Haring et l'héritage de la peinture américaine du XXème siècle
}

Hélène Gaillard

\section{(2) OpenEdition}

\section{Journals}

\section{Electronic version}

URL: http://journals.openedition.org/narratologie/7399

DOI: 10.4000/narratologie.7399

ISSN: 1765-307X

\section{Publisher}

LIRCES

\section{Electronic reference}

Hélène Gaillard, « Keith Haring et l'héritage de la peinture américaine du XXème siècle ", Cahiers de Narratologie [Online], 29 | 2015, Online since 08 January 2016, connection on 19 April 2019. URL : http://journals.openedition.org/narratologie/7399; DOI : 10.4000/narratologie.7399

This text was automatically generated on 19 April 2019

Article L.111-1 du Code de la propriété intellectuelle. 


\title{
Keith Haring et l'héritage de la peinture américaine du XXème siècle
}

\author{
Hélène Gaillard
}

1 S'il est communément accepté que Keith Haring (1958-1990) fut l'un des pionniers du street art, les sources de l'artiste américain sont trop souvent réduites au mouvement Pop art et au travail des graffeurs new-yorkais. Pourtant, Keith Haring reconnaissait avant tout l'influence de The Art Spirit écrit en 1923 par le peintre américain Robert Henri' ${ }^{1}$. C'est dans ce traité sur l'art que le chef de file de l'Ashcan school (c.1900-1915) définit l'artiste comme un élément s'inspirant du travail de ses prédécesseurs et de son environnement proche afin de rendre hommage à la vie et à l'ensemble de la communauté.

2 S'affranchissant peu à peu des tendances européennes, la peinture américaine au tournant du XXème siècle répond à une même volonté de redéfinir le rapport entre l'artiste et son public et d'interroger la notion d'espace artistique. Rejetant l'académisme et l'élitisme, l'art de cette nation bâtie sur un rêve démocratique se veut toujours plus proche du peuple et accessible au plus grand nombre. Annoncé par l'influent Robert Henri dans son ouvrage The Art Spirit, cet effort culmine avec le street art apparaissant à New York dans les années 80. En tant que pionnier de ce mouvement adepte du manifeste de Henri, Keith Haring inscrit alors le street art dans la continuité des écoles artistiques américaines $\mathrm{du}$ XXème siècle. Loin de se poser en rupture avec les pratiques préexistantes, le street art tel que conçu par Haring reprend les principes des grandes tendances picturales depuis le réalisme urbain jusqu'à l'expressionnisme abstrait en passant par le régionalisme.

3 A travers la résonance des courants artistiques du XXème siècle dans la pratique de Keith Haring, cette étude aura pour objectif de replacer les origines du mouvement street art au cœur d'une tradition américaine centrée sur le lien entre l'artiste et son public. Ce nouvel éclairage permettra de redéfinir les contours du street art en gardant à l'esprit la question du radicalisme de ce mouvement qui, parti d'une volonté de s'éloigner du musée, s'est progressivement institutionnalisé. 


\section{L'école de la poubelle : l'art descend dans la rue}

L'art descend dans la rue dès le tournant du XXème siècle, du moins thématiquement. Si l'on considère les principes régissant l'école de la poubelle dirigée par Robert Henri (1865-1929), il devient évident que ce courant annonce déjà le street art si tant est qu'il n'en est pas un. Peindre en rupture avec l'académisme, si possible en une minute ${ }^{2}$ afin de rester fidèle à son expression personnelle et pour célébrer la vie et l'homme sans distinction de classe : tel était le credo des artistes de l'Ashcan school que l'on pourrait aisément assigner aux street artists. On comprend alors pourquoi l'ouvrage de Henri se relève si précieux pour Haring. Dès 1904, Henri et ses confrères parmi lesquels John Sloan, William Glackens et George Luks exposent leurs travaux en dehors du circuit académique au National Arts Club mais c'est en 1908 que les huit artistes ${ }^{3}$ surnommés les « apôtres du laid» font sensation à la galerie Macbeth. Tels les graffeurs et les artistes urbains des années 70-80, ces réalistes modernes, opposés à l'élitisme et aux rigides conventions académiques, cherchent un nouvel espace pour exposer leur art rebelle. S'ils exposent hors des musées, ils sont aussi les premiers à investir le métro, non pas pour y réaliser directement des œuvres comme le fera Haring avec ses dessins à la craie, mais pour faire de cet espace typiquement urbain un sujet enfin digne de représentation. Avec Sixth Avenue Elevated After Midnight (1899) de Everett Shinn et Six O'clock, Winter (1912) peint par Sloan, les réalistes du début de siècle explorent le potentiel de ce lieu de transit et de regroupement où l'usager peut devenir plus aisément acteur et spectateur.

5 Bien que leur chef de file, Robert Henri, soit à cette époque membre de la National Academy of Design, ses œuvres dépeignant des scènes de la rue sont rejetées. Des sujets plus nobles à l'instar des tableaux de la haute société peints par John Singer Sargent sont en ce temps appréciés par l'institution artistique américaine. Avec des scènes incluant immigrants et vagabonds dans des environnements citadins souvent lugubres, les réalistes urbains font figure de révolutionnaires et sont aux antipodes des goûts conservateurs académiques. La presse ne manque pas de noter le courage de ces résistants dans cette guerre picturale ${ }^{4}$. En tant qu'anciens illustrateurs pour différents magazines, les Philadelphia Five du cercle de Henri sont proches des médias et savent les utiliser à bon escient, tout comme le fera des décennies plus tard Keith Haring dans ses entrevues télévisées. En traitant de sujets urbains, ces artistes se rapprochent des classes sociales les plus pauvres, ce qui leur vaut ainsi le titre de premiers artistes démocratiques. Avec Eva Green (1907), Robert Henri dépeint une jeune fille noire avec dignité et simplicité, déjouant ainsi les préjudices raciaux et les codes de l'art du portrait. Icône de l'école de la poubelle, The Spielers (1905) représente deux jeunes immigrées dansant fièrement joue contre joue dans un décor sombre et brumeux, ce qui renforce le contraste entre joie de vivre et misère. Cet appel à la fraternité, à la tolérance et à l'optimisme préfigure la même force expressive des dessins de figures dansantes de Haring ${ }^{5}$. Toutefois, l'engagement du cercle de Henri pour les plus mal lotis reste encore à apprécier. Issus de milieux plus aisés, ils se définissent comme de simples observateurs, témoins des multiples expériences de la vie contenues dans la vibrante cité de New York. Contrairement aux réalistes des années de dépression tels que Ben Shahn ou Raphael Soyer, il n'y a que rarement ${ }^{6}$ chez les peintres de l'Ashcan school de véritable souci de dénoncer l'injustice sociale. Leur champ d'application n'est pas limité à la misère urbaine, ils dépeignent le pire comme le meilleur de la vie citadine et s'intéressent aussi au monde 
bourgeois et aux loisirs. Mais, c'est précisément dans ce domaine que leur art présage le street art tel qu'initié par Haring.

6 Si Henri prône une exécution aussi rapide qu'en une minute, c'est parce qu'il s'agit de saisir sur l'instant l'énergie de la ville et de ses habitants. Au service de la vie ${ }^{7}$, l'art de la poubelle entend célébrer l'existence à travers la peinture de rues animées où chaque être est dépendant de l'autre. Cette vision à l'œuvre dans Hester Street (1905) de Luks ou dans Cliff Dwellers (1913) de Bellows sera reprise par Haring dans ses représentations plus stylisées de l'interconnexion humaine. Spectacle urbain d'une foule bigarrée et agitée, la fresque Tuttumondo (1989) réalisée à Pise amplifie et rend de manière plus abstraite les principes régissant la production des scènes de rue de Luks. Dynamisme, interaction et rayonnement constituent les clés de ces œuvres distantes de plus de soixante-dix ans. Le dynamisme se traduit par le flou du trait impressionniste chez les réalistes tandis qu'il est marqué par des doubles voire triples traits signifiant le mouvement et des lignes courbes continues par le street artist. Le rayonnement constitue aussi l'un des principes fondamentaux des deux arts : effet essentiel dans le compositions des réalistes comme vu dans Dutch Joe (1910) de Henri, il trouve son expression la plus vive dans l'image du radiant baby devenu l'une des signatures de Haring. Il faut noter que le recours à des sujets jeunes correspond à une volonté d'associer à l'énergie de la vie l'innocence de l'enfance. L'influence de Henri sur Haring peut encore se voir dans leur approche du monde de l'enfant. Pour le chef de file des réalistes, les enfants ont une grandeur qui fait d'eux un thème de prédilection: "If one has a love of children as human beings and realizes the greatness that is in them, no better subjects for painting can be found ${ }^{8}$ ». Haring confirme l'importance cruciale des enfants dans un art dédié à la vie ordinaire : " Children are the bearers of life in its simplest and most joyous form. When I do drawings with or for children, there is a level of sincerity that seems honest and pure $^{9}$. " Henri trouve en eux une source d'inspiration sans égale et une large part de ses toiles est centré e sur de jeunes sujets. Quant à Haring, il travaille directement avec des enfants et des adolescents sur plusieurs projets notamment sur Liberty Banner. Il réalise de nombreuses œuvres avec le graffeur LA II, alors âgé de seulement 14 ans, dont il admire le talent et la spontanéité. En quête d'authenticité et de spontanéité, Henri et Haring trouvent matière à travailler auprès d'un jeune public pour redéfinir la gamme d'objets d'étude mais aussi le statut d'artiste.

7 Inspiré par cette innocence et prônant la tolérance, ni le cercle de Henri ni les précurseurs du street art ignore cependant la question de la violence qui demeure inéluctable dans la communauté urbaine. Conséquence directe de la vie citadine où règnent promiscuité et agitation, la violence prend différentes formes selon qu'elle est représentée par les peintres de la poubelle ou le street artist. Elle est d'abord violence liée à l'injustice sociale chez Shinn dans Eviction (1904) qui dépeint la conséquence d'un acte condamnant une famille à l'insécurité de la rue. Haring fera écho à cette dimension en mettant en scène de multiples fois la violence générée par le racisme ou la drogue. Les réalistes exploitent aussi le potentiel pictural des rixes dans Street Fight (1910) peint par Shinn ou dans Curb Exchange (1907) de Glackens présentant à la fois les opposants mais aussi la foule de badauds. La force iconique de la violence mènent les réalistes et Haring à considérer celle-ci comme spectacle de vie. Ancien élève de Robert Henri, George Bellows peint selon les préceptes de l'école de la poubelle et fait figure de pionnier dans sa représentation de l'univers des combats de boxes. Inhérente à l'homme, la violence fait partie de l'identité masculine dans les célèbres Stag at Sharkey's (1909) ou Club Night (1909) 
de Bellows. Haring considère précisément cet aspect de la nature humaine dans son portrait du boxeur Macho Camacho (1985) en usant de son vocabulaire simple et précis pour figurer la violence comme le péché originel de l'homme : la jambe du sportif prend la forme d'un serpent la gueule ouverte, prêt à attaquer le boxeur. Ce vice humain se révèle prédominant dans l'environnement urbain des années 1910 et celui des années 1980 comme en atteste les scènes de foules peintes par les réalistes et les représentations plus apocalyptiques de Haring dans lesquelles la violence régit les rapports humains. Le traitement de ce sujet à travers les dessins faits dans le métro, c'est-à-dire là où le problème surgit, s'apparente à une mise en abîme. Cet effet concerne aussi les peintures de Bellows qui représentant le milieu clandestin de la boxe, sont exposées en marge de l'académie.

L'influence majeure de l'Ashcan school sur le street art réside bien dans cette célébration de la vie qui inclue le pire comme le meilleur de ce que la ville peut offrir. Une telle opération ne peut avoir lieu à cette époque qu'en dehors de l'institution muséale. La période de grande dépression (1929-1940) aux Etats-Unis rend plus pressant encore le besoin de trouver un nouvel espace artistique. Cette nécessité de se rapprocher toujours plus du peuple prépare le terrain pour les street artists.

\section{Le régionalisme : du musée au mur}

9 La conviction exprimée par Haring lorsqu'il affirme "Art is for everybody ${ }^{10}$ " prend racine pendant la période d'entre-deux-guerres. La dépression entraine la fermeture de nombreuses galeries et de grandes difficultés pour les musées en perte massive de visiteurs. Par ailleurs, la crise qui affecte toutes les classes sociales engendre une crise de confiance politique et une remise en cause de l'identité américaine. L'administration Roosevelt lance alors le New Deal qui inclue un projet dédié à l'art pour fournir d'abord du travail aux artistes mais aussi afin de s'adresser à la population. Sous l'égide du Federal Art Project ${ }^{11}$, l'art passe du musée au mur. Ce sont plus de 5000 œuvres murales qui sont produites en cette période afin de faire (re)-naître, à l'instar de Rivera au Mexique, un sentiment patriotique. C'est dans ce contexte que se développe une vive critique du musée comme lieu d'un art mort. Figure dominante du régionalisme et de l'art mural, Thomas Hart Benton compare le musée à un cimetière et entend replacer l'art dans des lieux de vie populaire tels que les saloons. Il affirme: "If it were left to me I wouldn't have have any museums...I'd like to sell \{my paintings\}to saloons bawdy houses, kiwanis and rotary clubs, chambers of commerce even women's clubs ${ }^{12}$ ». Cinquante ans plus tard, Haring déplore toujours la rigidité de l'institution qui ne s'ouvre pas à l'ensemble des classes sociales mais qui tire cependant avantage de la popularité de son travail : "It's really funny to me how all these museums sell poster and postcard reproductions of my art, but refuse to exhibit, collect or even acknowledge it within the museum ${ }^{13}$ ». Il évoque également la même nécessité de désacraliser l'art et de le rendre au peuple : «taking \{art\} off the pedestal...giving it back to the people ${ }^{14}$ » et travaille, tout comme les régionalistes, sur des projets commissionnés par diverses institutions pour exposition dans l'espace public.

Dans leur volonté de rendre l'art accessible à tous, les régionalistes cultivent un style coloré aux formes souvent arrondies sans véritable effet de profondeur et développent une esthétique de l'optimisme qui célèbre les valeurs du travail et du sens communautaire. Bien qu'ils peignent la campagne du Midwest plutôt que la grande ville, 
les trois maîtres régionalistes et leur style singulier annoncent le langage créé par Haring dans les années 80. Peuplée d'animaux, de simples fermiers et de figures de divertissement, l'œuvre de Curry préfigure le travail de Haring à bien des égards : tout ce monde s'y côtoie dans des scènes tantôt paisibles, tantôt cataclysmiques. L'inclusion d'éléments folkloriques récurrente dans les fresques de Benton - qui fait de son œuvre un art résolument populaire - constitue une stratégie réemployée par le street artist. Qu'ils s'agissent des régionalistes ou de Haring, il convient de voir par delà cette naïveté apparente car bien souvent, comme l'énonce Fabrice Hergott, « l'image d'un art coloré et joyeux, aux couleurs fluorescentes, en a donné la fausse image d'un art à destination des enfants - sinon enfantin ${ }^{15}$ ». Au delà de la simple référence à Mickey, à Cruella de Vil pour Haring ou à Huckleberry Finn ou à Frankie et Johnny pour Benton, on peut lire une certaine ironie dans la référence à des icônes populaires : l'appel à la tolérance prônée par Huck Finn et son ami Jim contraste avec la scène de lynchage peinte juste à coté. Quant à la célèbre souris de Walt Disney, Haring la représente telle une figure malicieuse envahissant les médias, hantant le drapeau américain et pesant sur l'humain malgré sa bonhommie. Derrière l'univers coloré et tout en rondeur des tableaux de Wood se joue un drame plus profond: celui de l'identité américaine dans American Gothic (1930) ou de la désertification des campagnes visible dans Fall Plowing (1931). Ainsi cette «naïveté géniale 16 » permet à Wood comme à Haring d'aborder des sujets sensibles tout en restant à la portée du grand public. Si l'on reprocha aux peintres de l'American Scene Painting leur affinité avec l'univers cartoonesque, c'est bien cette alliance subtile entre art populaire et discours protestataire, présente aussi chez Haring, qui permet de traiter de sujets sensibles en désamorçant l'impact polémique.

11 Certaines œuvres révèlent toutefois une vision clairement utopique : Benton dépeint dans America Today (1930) des scènes sans précédent à cette époque où travailleurs blancs et noirs travaillent de concert. Ces images préfigurent les messages de solidarité portés par des pyramides humaines et des cœurs dessinés sur des poitrines d'hommes. Visant à rassembler le peuple autour de l'art, Benton se spécialise dans des fresques figurant l'interconnexion humaine : qu'il s'agisse de lien historique, d'une union autour de loisirs ou encore d'un groupe de travailleurs, chaque homme est lié à son prochain. C'est cet effet qui conduira de nombreux critiques à qualifier de démagogie picturale ce profond $\mathrm{d}$ ésir de s'adresser au peuple. En quête d'authenticité et porteur de vérité sociale, les régionalistes sont cependant aussi parfois polémiques comme en atteste la critique du travail de Curry pour le capitole du Kansas. Son choix de peindre au centre de l'une de ses fresques le très controversé John Brown ${ }^{17}$, qualifié de terroriste pour certains et de martyr pour d'autres, cause à Curry de nombreux problèmes et l'artiste ne signa d'ailleurs jamais son travail. Proche du peuple, l'art des années 30 comme celui des années 80 une affaire publique et tous deux incluent inexorablement des sujets de leur temps comme le soutient Haring: " an artist is a spokesman for a society at any given point in history ${ }^{18}$ ». Néanmoins par la subtilité de leur style innocent, le régionalisme et le travail de Haring apparaissent plus populaires que contestataires. L'objectif premier semble dans les deux cas d'informer et d'unir plus que d'inciter à la rébellion. Impliqué contre l'Apartheid, la drogue et le sida et ardent défenseur de l'écologie, Haring prend part à de nombreuses luttes mais fait peu souvent l'objet de controverse. L'un des combats communs aux artistes que près de cinquante ans séparent est l'avancée industrielle incontrôlée et ses répercussions sur la culture commune. Benton aborde le sujet dans A Social History of the State of Missouri (1936) où l'épaisse fumée noire se dégageant des trains et des usines semble un danger menaçant l'équilibre naturel. Pour 
Benton, la technologie peut apporter le progrès si elle intègre l'homme et la nature comme le prouve ses images où machines et plantes sont entremêlées. Conscient lui aussi des problématiques liées au progrès, Haring emploie, dans The Last Rainforest (1989) parmi d'autres exemples, une iconographie apocalyptique propre à l'ère technologique faite de bombes atomiques, de robots et de soucoupes, telle une mise en garde contre un mauvais usage des découvertes.

Enfin ce qui unit surtout Haring à l'héritage régionaliste est le lien étroit entre art et commerce, conséquence du souhait d'accessibilité au plus grand nombre. Benton et ses confrères recourent à de nouvelles techniques de reproduction et de diffusion et ouvrent ainsi une voie que suivra Haring avec son pop shop. Collaborant avec les magazines Time et Life, Benton et Curry participent à des campagnes de publicité pour des sociétés qui, à l'instar de Lucky Strike, solliciteront des décennies plus tard Haring. En effet, la simplicité des messages caractérisant leur esthétique vernaculaire en fait des représentations idéales pour la publicité. Liés à l'Associated American Artists, une entreprise privée créée en 1934 qui vend des gravures et des lithographies de pièces originales pour seulement cinq dollars, Benton, Wood et Curry concrétisent leur ultime dessein en défiant l'idée que l'art n'est destiné qu'aux élites. "Original artworks which once only museums and the rich could afford are now yours to live with at home ${ }^{19} »$ : cette idée soutenue par le AAA est reprise par Haring lorsqu'il ouvre en 1986 son premier Pop Shop à Soho. Loin d'être une entreprise rentable, le pop shop relève de cet effort continu vers une démocratisation de l'art. Cherchant à sortir des sentiers étroits du marché de l'art, Haring conçoit sa boutique comme l'extension de son art :

There's the philosophy behind the Pop Shop: I wanted to continue the same sort of communication as with the subway drawings. I wanted to attract the same wide range of people and I wanted it to be a place where, yes, not only collectors could come, but also kids from the Bronx ... this was still an art statement ${ }^{20}$.

13 Si de nombreux aspects rapprochent les régionalistes de Haring, une différence fondamentale réside dans l'absence d'ambition internationale chez les artistes de l'entredeux-guerres. Apres la seconde guerre mondiale, l'art évolue vers une dimension universelle et redéfinit ses voies d'expression et de communication. Face à un monde de plus en plus complexe et avancé technologiquement pouvant être anéanti par la puissance atomique, l'art aux Etats-Unis se fait plus primitif et les artistes sont en quête de nouveaux modes d'expression. Jackson Pollock confirme ce besoin lorsqu'il propose : « the modern painter cannot express his age, the airplane the atom bomb, the radio, in the old forms of Renaissance or of any past culture. Each age finds its own technique ${ }^{21}$ ».

\section{L'Action painting : un langage de signes spontané}

Un des peintres que cite à plusieurs reprises Haring dans son autobiographie est justement Jackson Pollock, précurseur de l'Action painting. Dans son autobiographie, le street artist affirme explorer les mêmes pistes que Dubuffet, Davies mais aussi que Pollock $^{22}$. Dans les années 1978 à 80, le style de Haring est très proche de celui de l'expressionniste abstrait, il peint des formes non figuratives sur une toile directement posée au sol. L'importance du geste chez Haring fait écho au modus operandi du leader de l'école de New York; l'une des particularités du travail du street artist reste l'absence totale d'esquisses ou de quelconques dessins préparatoires. 
Cette spontanéité place Haring dans la lignée de Pollock et de son approche directe : «I approach painting the same way I approach drawing, that is direct - with no preliminary studies $^{23}$ ». La ligne se veut continue, seulement interrompue pour tracer une figure non adjacente pour Haring. La continuité chez Pollock est exprimée à travers la régularité des applications de pinceaux à un rythme effréné donnant l'impression d'une trance picturale. A l'instar de Pollock, Haring peint d'une seule traite, sans pause ni esquisse. Vers la fin de sa carrière, le street artist privilégie les enchevêtrements plus denses qui cèdent d'autant plus place au geste pictural. Le choix de matériaux peu conventionnels sur des supports aux dimensions monumentales singularisent leur procédé artistique : rompant avec la tradition de l'huile, Pollock se tourne vers la peinture acrylique industrielle qu'il applique bien souvent à l'aide de simples bâtons en guise de pinceaux. Haring recherche également de nouvelles pistes d'expression : il considère la simplicité de la craie, idéale bien qu'éphémère sur les panneaux noirs pour ses dessins dans le métro. Il s'essaie aussi à différentes encres dont le sumi, mélange de suie et de colle pour ses œuvres réalisées en intérieur. Il opte finalement pour la peinture vinylique appliquée sur des bâches de vinyle car l'élitisme de la toile bloque sa créativité et l'inhibe : «Canvas as a material is wonderful. It is sturdy and can be sold and is somewhat permanent. But I am inhibited by $i^{24}{ }^{24}$. Pollock avait déjà entamé pareil processus de désacralisation de la toile en lui ôtant sa suprématie murale et en la posant au sol libre, non tendue et sans apprêt: "My painting does not come from the easel. I hardly ever stretch my canvas before painting. I prefer to tack the unstretched canvas to the hard wall or the floor. I need the resistance of a hard surface ${ }^{25} »$. De l'ensemble de ces spécificités techniques résulte le dripping, signature de Pollock que l'on retrouve aussi chez Haring. Garantissant fluidité, liberté et le dynamisme tant recherche, la coulure est la trace de l'acte de peindre et atteste de l'immersion totale de l'artiste dans son œuvre. Tel un défi à la précision et à la maitrise atteintes par les grands maitres, le drip marque aussi une certaine décadence, apanage d'un monde moderne corrompu. Vers la fin de sa vie alors qu'il se sait malade du sida, Haring accentue cet effet visible dès le début de sa carrière en appliquant sur des contours déjà tracés de brefs coups de pinceaux : la surface picturale est ainsi marquée de coulées qui suggèrent la déliquescence de la société et la dégénérescence de son propre corps.

16 Plus que des techniques semblables, c'est le principe qui régit leurs arts qui rapproche encore les deux Américains. Le tracé continu sans préparation répond au désir de liberté ; loin de la rigueur et du contrôle académique, il laisse l'inconscient, la nature véritable de l'artiste s'exprimer. Il y a, chez Pollock comme chez Haring, une volonté de dominer l'espace pictural et de faire corps avec la toile. Pollock exprime ce besoin en ces termes: " On the floor I am more at ease. I feel nearer, more a part of the painting, since this way I can walk around it, work from the four sides and literally "be" in the painting ${ }^{26} »$. Dans le New-York des années 80 divisé en ghettos et en quartiers qui ne connait que peu de mixité sociale, Haring entend reprendre contrôle de l'espace artistique et urbain en se confrontant au lieu :

Possibly the reason I insist on spending the first few minutes of a painting drawing a border around the area I am about to paint is because I am familiarizing myself with the scale of the painting I am about to paint. I am physically experiencing the entire perimeter of a given space ${ }^{27}$.

17 La pose qu'il prend dans son pop shop est particulièrement révélatrice : Haring, nu et couvert de peinture, se fond dans son œuvre, il est l'œuvre et dépasse alors l'idéal de Pollock qui affirmait être dans la peinture. C'est en s'ouvrant à la surface à peindre que 
tous deux parviennent à laisser libre cours à l'improvisation et à la réalité intérieure comme l'affirme Pollock: "The modern artist, it seems to me, is working and expressing an inner world - in other words - expressing the energy, the motion and the other inner forces... The modern artist is working with space and time, and expressing his feelings rather than illustrating ${ }^{28}$ ». Dans le street art, le moi intérieur est d'autant plus extériorisé qu'il appartient strictement au domaine extérieur, à la rue. Fait de gestes et de formes simples, le langage esthétique de Pollock et celui de Haring résultent d'une réalité psychologique intérieure qui renoue avec la nature primitive de l'homme.

18 Fasciné par les cultures amérindiennes, Pollock s'exprime dans un langage universel où les lignes et la couleur font office de grammaire et de vocabulaire. Lorsqu'il affirme que son procédé est proche de celui utilisé par les Indiens de l'ouest pour dessiner dans le sable, il révèle que chacun des signes de ses œuvres font sens. Accomplie par la spontanéité du geste, cette langue s'adresse à tous et ne nécessite aucune connaissance particulière pour faire sens. En cela, son travail artistique appartient au champ primitif. Intégrant des éléments figuratifs, The She-Wolf (1943) est composé de formes géométriques et de signes dont la lecture, affirme Pollock, ne peut être effectuée que par le spectateur: "Any attempt on my part to say something about it, to attempt explanation of the inexplicable, could only destroy $\mathrm{it}^{29}{ }^{29}$. En visant l'universalité par le sensible, Pollock accomplit un retour à l'essence même de l'homme et établit de nouveaux mythes modernes pouvant s'adresser à tous. C'est un but semblable que veut servir quarante ans plus tard Haring en recourant majoritairement à des couleurs primaires et en utilisant un langage de pictogrammes. S'il s'oppose à la notion de primitivisme, il reconnait que son travail est dicté par une sensibilité intérieure. Pendant sa formation à la New York School of Visual Arts, Haring étudie la sémiotique et, marqué par les écrits de Roland Barthes, il interroge la relation entre signifiant et signifié : «I am intrigued with the shapes people choose as their symbols to create a language ${ }^{30} »$. Il développe alors un alphabet semi-abstrait, accessible à tous, sans jamais vraiment en révéler le sens car il appartient au public de définir son œuvre. Selon Haring, tel est le devoir du spectateur : « It is the responsibility of the viewer or interpreter who will receive my information to derive their own ideas or meanings from $i^{31}{ }^{31}$. C'est dans le même esprit qu'à l'instar de Pollock, il intitule peu souvent ses réalisations. Haring fait de sa grammaire picturale un système capable de transcender les capacités expressives des autres codes écrits. Certaines icônes récurrentes telles que les cœurs ou les sexes en érection pour les plus simples et les croix ou les abdomens perforés pour les plus ambigus, fonctionnent à la manière de hiéroglyphes : ils peuvent représenter directement une chose ou une notion mais peuvent aussi renvoyer à un sens plus arbitraire selon le contexte. Dans d'autres œuvres, la répétition de signes ou la saturation de symboles forcent l'attention du spectateur qui doit les décrypter. Inscrits sur l'espace public, ces signes qui précèdent toute communication écrite renvoient aux dessins rupestres de l'ère préhistorique mais aussi aux pratiques des artistes de l'école de New York. Le style de Haring emprunte à Pollock et à son épouse Lee Krasner un langage fait d'icônes miniatures et de petites formes abstraites interconnectées. Son art fait de Haring un scribe de l'ère postmoderne moins mystique que ses prédécesseurs mais tout aussi conscient du besoin d'un nouveau mode d'expression. 


\section{Conclusion}

19 l'apport des réalistes, des régionalistes et des expressionnistes, l'œuvre de Haring aurait sans doute pris une autre forme. Si le street artist partage l'idée de Henri que tout artiste, forcement inspiré par ses prédécesseurs, ne doit pas chercher à imiter mais proposer sa propre vision du monde, sa dette envers les générations passées d'artistes américains repose essentiellement sur cette ouverture et destination de l'art au grand public. Comme nombres de peintres du XXème siècle, Haring était en quête de signes directement accessibles aux masses, puisant ainsi dans le répertoire de ses prédécesseurs pour développer son vocabulaire graphique. Héritier de Henri, de Benton et de Pollock, il a à son tour ouvert la voie en démocratisant non seulement l'art mais aussi l'espace artistique.

Sa volonté de reconnaissance en tant qu'artiste par l'institution muséale pourrait être perçue comme un paradoxe, voire une trahison des idéaux auquel son œuvre se rattache. Ce sont ses subway drawings, éphémères et anonymes, qui lui ont donné accès à la notoriété mais sa reconnaissance en tant qu'artiste reste tributaire de l'appréciation institutionnelle. Cependant, en multipliant les espaces de visibilité de l'art et en instituant le pop shop comme un de ses nouveaux lieux, Haring a établi un véritable lien entre musées et murs et a redonné un nouveau souffle à l'attraction artistique même si ceci passe inexorablement par la marchandisation de l'œuvre. L'exemple de Haring et de ses influences permet de mieux cerner les ambivalences toujours décelables au coeur du street art, une pratique artistique populaire, à la frontière entre espace public et domaine privé et dont la valeur éphémère est pourtant soumise à une dynamique de marché.

\section{BIBLIOGRAPHY}

Baigell, M. (1984) A Concise History of American Painting and Sculpture. New York, Harper\&Row.

Blinderman,B. (1992) Keith Haring : Future Primeval. New York: Abbeville Press.

Doss, E. (1991) Benton, Pollock and the Politics of Modernism : from Regionalism to Abstract Expressionism. Chicago: Chicago Press.

Doss, E. (2002) Twentieth Century American Art. Oxford: Oxford University Press.

Drenger, D. (1988) « Art and Life : An Interview with Keith Haring », in Columbia Art Review, 44-53.

Gruen,J. (1991) Keith Haring : The Authorized Biography. New York : Simon\&Schuster

Haring, K. (1986) Keith Haring Journals. New York : Penguin Books.

Henri, R. (1923) The Art Spirit. New York : Harper\&Row.

Cahiers de Narratologie, 29 | 2015 
Hergott,F. (2013) Avant-propos du catalogue d'exposition in Keith Haring, The Political Line. Paris: Paris Musées, 11-13.

Hoopes, D. (1976) Robert Henri. New York : Chapellier Galleries.

Karmel, P. (1999) Jackson Pollock : Interviews, Articles, and Reviews. New York : The Museum of Modern Art.

Kolossa,A.(2009) Keith Haring. Koln :Taschen.

Leja, M. (1993) Reframing Abstract Expressionism : Subjectivity and Painting in the 1940s. New Haven : Yale University Press.

Ross, C. (1990) Abstract Expressionism : Creators and Critics. New York : Abrahams Publishers.

\section{NOTES}

1. "It was almost a bible to me ", Keith Haring cité par Barry Blinderman, Keith Haring: Future Primeval, New York, Abbeville Press, 1992, p.95

2. « Work with great speed. \{...\} Do it in one minute if you can », Robert Henri, The Art Spirit, New York, Harper\&Row, (1923) 1984, p.26.

3. Parmi les huit artistes, on compte cinq réalistes urbains (Robert Henri, George Luks, William Glackens, John Sloan et Everett Shinn) et trois autres peintres (Arthur B. Davies, Ernest Lawson et Maurice Prendergast) ayant participé à l'exposition mais dont les styles et les sujets sont sensiblement différents.

4. Un article paru le 2 février 1908 dans The New York World fait état du radicalisme des artistes en les décrivant tels des rebelles «osant " peindre la ville de New York. (cf. Erika Doss, Twentieth Century American Art, Oxford, Oxford University Press, 2002, p. 37)

5. A ses débuts, le street art est proche du mouvement hip-hop. Inspiré par les break dancers et le milieu Electric Boogie qu'il fréquente, Keith Haring rend hommage aux liens unissant arts visuels et arts vivants dans nombres de ses œuvres.

6. Matthew Baigell note à ce propos : «less concern with social problems than their subject matter might indicate, the artists revealed more in their responses to life than in the kind of life they found during their visits to the ghetto districts. " cf. Matthew Baigell, A Concise History of American Painting, New York, Harper\&Row, 1984, p. 197. Everett Shinn est sans doute le plus socialement engagé avec des scènes d'expulsion et de misère sociale.

7. L'une des plus célèbres formules de Henri est celle de "Art for Life's sake " qui, opposée à l'idée de "l'art pour l'art », s'éloigne d'un intérêt purement esthétique pour considérer une célébration artistique de la vie.

8. Robert Henri cité par Donelson F. Hoopes, Robert Henri, New York, Chapellier Galleries, 1976, n.p.

9. Keith Haring, Keith Haring Journals, New York, Penguin Books (version numérique), 1986, p.228.

10. Keith Haring, op.cit., p.82.

11. Lancé par Roosevelt dans le cadre du Work Progress Administration, le Federal Art Project a fonctionné d' août 1935 jusqu'à juin 1943 et a financé plus de 200000 œuvres. cf. Francis O'Connor, Federal Support for the Visual Arts : the New Deal and Now., Greenwich, New York Graphic Society, 1969.

12. Thomas Hart Benton cité par Erika Doss, Benton, Pollock and the Politics of Modernism : from Regionalism to Abstract Expressionism, Chicago, Chicago Press, 1991, p.281.

13. Keith Haring, op. cit., p. 377.

14. Daniel Drenger, "Art and Life: An Interview with Keith Haring ", Columbia Art Review, Printemps 1988, p.53. 
15. Fabrice Hergott, Avant-propos du catalogue d'exposition Keith Haring, The Political Line, Paris Musées, Paris, 2013, p. 12.

16. Alexandra Kolossa, Keith Haring, Köln,Taschen, 2009, p.28.

17. Ardent défenseur de l'abolition de l'esclavage engagé dans une lutte armée, John Brown (1800-1859) entreprit de soulever les esclaves de Virginie et de leur fournir des armes par la prise de l'arsenal fédéral de Harpers Ferry. Son exécution eut un retentissement considérable sur l'opinion mondiale en faveur du mouvement abolitionniste.

18. Keith Haring, op.cit, p.209.

19. Communiqué du AAA cité par Erika Doss, Benton, Pollock and the Politics of Modernism: from Regionalism to Abstract Expressionism, Chicago, Chicago Press, 1991, p.161.

20. Keith Haring cité dans John Gruen, Keith Haring: The Authorized Biography, New York, Simon\&Schuster,1991, p.148.

21. Jackson Pollock dans un entretien en 1950 cité par Erika Doss, op.cit., p. 331.

22. Haring écrit dans son journal : "I hope I am not vain in thinking that I may be exploring possibilities that artists like Stuart Davis, Jackson Pollock, Jean Dubuffet and Pierre Alechinsky have initiated but did not resolve. » op.cit., p. 97.

23. Jackson Pollock cité par Michael Leja, Reframing Abstract Expressionism : Subjectivity and Painting in the 1940s, New Haven, Yale University Press,1993, p.186.

24. Keith Haring,op.cit., p.87.

25. A la demande de Robert Motherwell, Jackson Pollock décrit sa méthode dans " My Painting ", Possibilities I, pp. 78-83, 1947 cité dans Pepe Karmel, Jackson Pollock: Interviews, Articles, and Reviews, New York, The Museum of Modern Art, 1999, p.17.

26. Ibid.

27. Keith Haring, op.cit., p.27.

28. Jackson Pollock dans un entretien avec William Wright, 1950 cité dans Clifford Ross, Abstract Expressionism : Creators and Critics, Abrahams Publishers, New York, 1990, p. 139.

29. Jackson Pollock cité dans Pepe Karmel, op. cit. p. 237.

30. Keith Haring, op.cit., p.120.

31. Ibid., p.121.

\section{ABSTRACTS}

Although Keith Haring is seen as a pioneer in the street art movement, the sources of his work are often limited to Pop Art. Yet, Haring's street art practices do not depart from the past trends retaining instead many key aspects and principles at the core of 20th century American art. As a matter of fact, the street artist acknowledges Ashcan school leader Robert Henri - who started a trend said to be the first democratic American art-as his main influence. By observing to what extent Haring's art echoes the preexisting American artistic values, this study will provide a new historic context to the origins of street art. It will also lead to reexamine the question of street art's radicalism, considering the gradual evolution of its paradoxical acceptance into museums.

Si Keith Haring est bien reconnu comme l'un des pionniers du street art, les sources de son travail sont trop souvent réduites au Pop art. Loin de se poser en rupture avec les pratiques préexistantes, le street art tel que conçu par Haring reprend les codes et la raison d'être des grandes tendances picturales du XXème siècle aux Etats-Unis. Le street artist cite d'ailleurs 
comme influence majeure Robert Henri, chef de file de l'école de la poubelle, un courant qui annonce la démocratisation de l'art américain. A travers la résonance des courants artistiques du XXème siècle dans la pratique de Keith Haring, cette étude aura pour objectif de replacer les origines du mouvement street art au coeur d'une tradition américaine centrée sur le lien entre l'artiste et son public et la notion d'espace artistique. Ce nouvel éclairage permettra de redéfinir les contours du street art en gardant à l'esprit la question du radicalisme de ce mouvement qui tend à s'institutionnaliser.

INDEX

Chronological index: XXe siècle

Mots-clés: street art, peinture, réalisme, régionalisme, expressionnisme abstrait, héritage, influence

Geographical index: Etats-Unis

\section{AUTHOR}

\section{HÉLÈNE GAILLARD}

Université Nice Sophia Antipolis, Laboratoire LIRCES 